Article

\title{
Expression of $A h D R E B 1$, an AP2/ERF Transcription Factor Gene from Peanut, Is Affected by Histone Acetylation and Increases Abscisic Acid Sensitivity and Tolerance to Osmotic Stress in Arabidopsis
}

\author{
Baihong Zhang ${ }^{1}$, Liangchen $\mathrm{Su}^{1,2}$, Bo $\mathrm{Hu}^{1}$ and Ling $\mathrm{Li}^{1, *}$ \\ 1 Guangdong Provincial Key Laboratory of Biotechnology for Plant Development, School of Life Sciences, \\ South China Normal University, Guangzhou 510631, China; zhangbyhome@126.com (B.Z.); \\ sksky163@163.com (L.S.); hubo0610@126.com (B.H.) \\ 2 Department of Bioengineering, Zhuhai Campus of Zunyi Medical University, Zhuhai 519041, China \\ * Correspondence: liling502@126.com; Tel.: +86-020-8521-1378
}

Received: 6 April 2018; Accepted: 9 May 2018; Published: 11 May 2018

\begin{abstract}
Drought stress negatively affects plant growth and development. An increasing number of reports have revealed the involvement of APETALA2/Ethylene Responsive Factor (AP2/ERF) transcription factors (TFs) in biotic and abiotic stress regulation in plants. However, research on these TFs in the peanut plant (Arachis hypogaea) has been limited. Here, we isolated a full-length coding sequence (CDS) of the AP2/ERF family gene AhDREB1 from the peanut plant and showed that its expression was induced by Polyethylene Glycol (PEG) 6000 and exogenous abscisic acid (ABA) treatment. When overexpressed in Arabidopsis, AhDREB1 increased both ABA levels and ABA sensitivity, affected the ABA signaling pathway and increased the expression of downstream drought stress-related genes RD29A, P5CS1, P5CS2 and NCED1. These results demonstrate that AhDREB1 can improve tolerance to drought via the ABA-dependent pathway in Arabidopsis. In the peanut plant, the specific histone deacetylases (HDACs) inhibitor trichostatin A (TSA) promotes AhDREB1 transcription and the enrichment level of $\mathrm{H} 3 \mathrm{ac}$ was increased in regions of the AhDREB1 gene during TSA and PEG treatment. In summary, histone acetylation can affect the expression of AhDREB1 under osmotic stress conditions, thereby improving plant drought resistance.
\end{abstract}

Keywords: APETALA2/Ethylene Responsive Factor; AhDREB1; peanut; abscisic acid; drought stresses; histone acetylation

\section{Introduction}

Plants are sessile and constantly subjected to various abiotic stresses, including drought, cold, high salinity, heat and alkalinity, which seriously affect their growth, development and productivity [1]. Of these stresses, drought is one of main causes of loss in production of agricultural crops across the world [2]. Drought can result in plant water loss and leaf wilting, and can lead to irreversible, damaging changes including, ultimately, plant death [3].

Over a long period of evolution, plants have developed diverse survival strategies and complex mechanisms to cope with drought stress [4]. Plants respond to drought stress by altering molecular and physiological processes at the molecular, cellular, tissue and whole-plant levels [5,6]. At the molecular level, plants can optimize their growth and development by regulating the expression of numerous stress responses via two main pathways, one of which is abscisic acid (ABA)-dependent, while the other is not [7]. However, several studies suggest that, in fact, there may be cross-talk between the two types of signaling pathway; thus, they may be interdependent [8]. The phytohormone ABA is a pivotal 
regulator of plant growth and development and of plant responses to drought stress [3]. The content of $\mathrm{ABA}$ in plants increases rapidly under drought stress conditions, and returns to its original level when the stress is removed. Because it correlates with drought resistance, the level of ABA is an important index of drought resistance in plants [9]. Multiple processes are involved in the biosynthesis of ABA, but the main factor during drought is the increase in expression of 9-cis-epoxycarotenoid dioxygenase (NCED), which encodes a key enzyme in the ABA biosynthetic pathway [10,11]. In addition, plants regulate the expression of drought-resistance genes via relevant components of ABA signaling pathway, thus protecting plants from drought stress. ABA can change the transcription level of drought-related genes via the ABA-responsive element (ABRE) binding protein [12,13].

In the ABA-independent pathway, dehydration-responsive element binding protein (DREB), plays the main role in regulating many drought-responsive genes. Other transcription factors such as NAC (NAM, ATAF, CUC), MYB (v-myb avian myeloblastosis viral oncogene homolog), MYC (Myelocytomatosis proteins), WRKY and nuclear factor-Y (NF-Y) are also involved in drought response and tolerance [14]. DREB activates drought-associated genes, such as RD29A, P5CS1, SODs, by binding to dehydration responsive element (DRE) motifs $[15,16]$, which comprise nine conserved bases (TACCGACAT), in their promoter regions, thereby increasing the plant's drought resistance [14,17]. DREB transcription factors form a subfamily of the APETALA2/Ethylene Responsive Factor (AP2/ERF) protein family $[18,19]$.

$\mathrm{AP} 2 / \mathrm{ERF}$ are a large family of plant-specific transcription factors (TFs) and the rapid development of genome-wide sequencing technology and bioinformatics tools has led to the identification of AP2/ERF genes in many species; for example, 139 and 122 AP2/ERF genes have been found in rice and Arabidopsis thaliana, respectively [20]. TFs from the AP2/ERF family have either one or two highly conserved DNA-binding AP2/ERF domains, each of 58 or 59 amino acids residues [21]. Based on DNA binding domain (DBD) and sequence similarity, AP2/ERF family members have been divided into four major subfamilies, comprising DREB factors, ERFs, AP2 factors and a subfamily called Related to ABI3 and VP1 (RAV) $[18,19]$. The DREB and ERF subfamilies, both containing a single AP2 domain, are distinguished from each other by differences in the conserved residues of their DBDs. In DREB proteins, the 14th and 19th positions of the DBD are valine (V14) and glutamic acid (E19), whereas in ERFs, the corresponding positions are alanine (A14) and aspartic acid (D19) [18]. Members of the AP2 subfamily have two AP2/ERF domains, while members of the RAV subfamily have a B3 DBD in addition to an AP2/ERF domain [18,19].

In recent years, an increasing number of reports have revealed AP2/ERF TFs to be involved in multiple biological processes that regulate plant growth and development, and especially in abiotic stress tolerance. Among the AP2/ERF family, DREB and ERF subfamily proteins have been well characterized in abiotic stress responses involving drought, salt, cold and other stresses [20]. Thus, overexpression of GsERF71, which encodes an AP2/ERF TF from wild soybean, improves plant alkaline stress tolerance by upregulating $\mathrm{H}^{+}$-ATPase and by modifying the accumulation of auxin [22]. In Arabidopsis, overexpressing AtERF019 increases tolerance to water deficiency, and also results in phenotypes that delay flowering and senescence [23]. In Arabidopsis, overexpression of DREB1 can enhance tolerance to cold stress, promote the accumulation of proline and result in phenotypes of plant $\mathrm{d}$ warfing and delay of flowering [24]. Overexpression of $S C D R E B 8$ significantly improves salt tolerance of Arabidopsis at the seedling stage by upregulating the expression of downstream stress-related genes and improving reactive oxygen species (ROS) scavenging ability [25]. Overexpression of OsDREB genes enhances drought tolerance in rice [26]. In Arabidopsis, strains overexpressing AtDREB2C are highly sensitive to ABA during seed germination and show enhanced tolerance to cold and heat stress [8].

It has been shown that epigenetic regulation plays an important role in plant growth and development [27]. Epigenetic regulation mainly involves two aspects of DNA and histone. At the level of DNA, it is mainly the methylation of genomic DNA, while at the histone levels are mainly histone acetylation, methylation, glycosylation, phosphorylation, ubiquitination, and so on [28,29]. Histone 
acetylation levels are controlled by the dynamic equilibrium between histone acetyltransferases (HATs) and histone deacetylases (HDACs) [30]. Thus, acetylation of histone lysine residues by HATs can relax chromatin structure and thus facilitate gene activation. Conversely, HDACs remove acetyl groups from acetylated histones, and this activity is generally associated with transcriptional repression and gene silencing [31]. Recently, some studies have shown that histone acetylation can affect the development of plant seeds, the formation and opening of flowers, the elongation of roots, gametophyte development and other biological processes. [32,33]. In maize, mutation of the HDAC gene hda101 results in a smaller kernel than that of wild type, and in defects of basal endosperm transfer layer (BETL) cells, which suggests that HDA101 can affect the development of seed in maize [34]. In A. thaliana, AtHDA5 affects flowering time, and the $h d a 5$ mutant exhibits a late-flowering phenotype [35]. An increasing number of reports have shown that histone acetylation affects $\mathrm{ABA}$ and other phytohormone signaling pathways, and is also important for the epigenetic regulation of plant responses to external stress [36,37]. However, the specific mechanisms are not clear. Recently, it has been reported that the MSI1-HDA19 complex affects ABA signaling pathway by binding to the chromatin of ABA receptor genes PYL2, PYL4 and PYL6, where it maintains low levels of acetylation of histone $\mathrm{H} 3$ at lysine 9, thereby regulating expression levels of these genes [38]. In A. thaliana, AtHDA9 negatively affects plant sensitivity to drought and salt stresses by modifying histone acetylation levels of a large number of stress-responsive genes [27]. In maize, HDACs can affect the level of histone acetylation in chromatin, which in turn promotes the expression of ZmDREB1 under cold stress [39].

Peanut (Arachis hypogaea L.) is one of the most important oil crops and food sources of protein, and is cultivated in the semi-arid tropical and subtropical regions of the world [40]. Peanut plants are constantly subjected to various biotic and abiotic stresses during growth and development. Drought stress is one of the main factors that limit the growth, yield and quality of peanuts [36]. Therefore, it is meaningful to study how peanut plants respond to drought stress, which has potential applications in the breeding of drought-resistant strains. Previously, in the peanut transcriptome database, we found a DREB-like gene (comp63385_c0) that contained a key AP2/ERF domain and was upregulated by water deficit and exogenous ABA [41]. This gene was named AhDREB1 (GenBank accession No. KU143745.1). In this paper, the full-length coding sequence (CDS) of AhDREB1 was isolated from peanut leaves and its transcription was found to be induced by Polyethylene Glycol (PEG) 6000 and exogenous ABA treatment. Overexpression of AhDREB1 in Arabidopsis increased ABA sensitivity during seed germination, affected the expression of $A B A$ signaling pathway-related genes and enhanced drought resistance. Furthermore, the H3ac enrichment level was increased in various regions of AhDREB1 chromatin during osmotic stress and treatment with the specific HDAC inhibitor trichostatin A (TSA). In summary, histone acetylation can affect the expression of AhDREB1 under drought conditions, and may be important in further improving drought resistance of peanut plants.

\section{Results}

\subsection{Isolation and Characterization of Peanut AhDREB1 Gene}

The full-length CDS of AhDREB1 gene was isolated from peanut leaf DNA consists of a 1050-bp open reading frame (ORF) that encodes a polypeptide of 349 amino acid residues and predicted molecular mass $\sim 37.8 \mathrm{kDa}$ (Figure 1A). Sequence analysis showed that AhDREB1 contains one conserved AP2/ERF DBD (residues 167-227), including the two conserved residues V14 and L19 specific to DREB TFs [18]. Multiple sequence alignment using DNAMAN8.0 software suggested that $A h D R E B 1$ also has a low degree of similarity to the known DREB genes from A. thaliana, Glycine max, Oryza sativa, Solanum lycopersicum and Zea mays outside the AP2/ERF domain (Supplementary Figure S1). The AhDREB1 promoter sequence contains many putative stress response-related cis-elements, including examples of the ABRE element (6 hits), CE3 recognition site (1 hit), MYB recognition site (1 hit) and heat shock response element (HSE) recognition site (3 hits) (Supplementary Figure S2). Secondary structure prediction suggested AhDREB1 has 
no transmembrane structure (Figure 1B). To determine the subcellular localization of AhDREB1, we engineered a plasmid construct in which the AhDREB1 sequence without the stop codon was inserted into the $5^{\prime}$ end of the eGFP gene in the p35S-eGFP vector. The p35S::AhDREB1-eGFP recombinant plasmid was transformed into Arabidopsis protoplast cells, with the empty p35s-eGFP vector used as a control. Confocal microscopy imaging showed that, while the eGFP (enhanced Green Fluorescent Protein) control protein was distributed throughout the cell, the AhDREB1-eGFP fusion protein was only visible in the nucleus (Figure 1C). These results suggest that $A h D R E B 1$ encodes a nuclear protein containing one conserved AP2/ERF DBD, and that AhDREB1 belongs to the DREB TF subfamily.

(A)
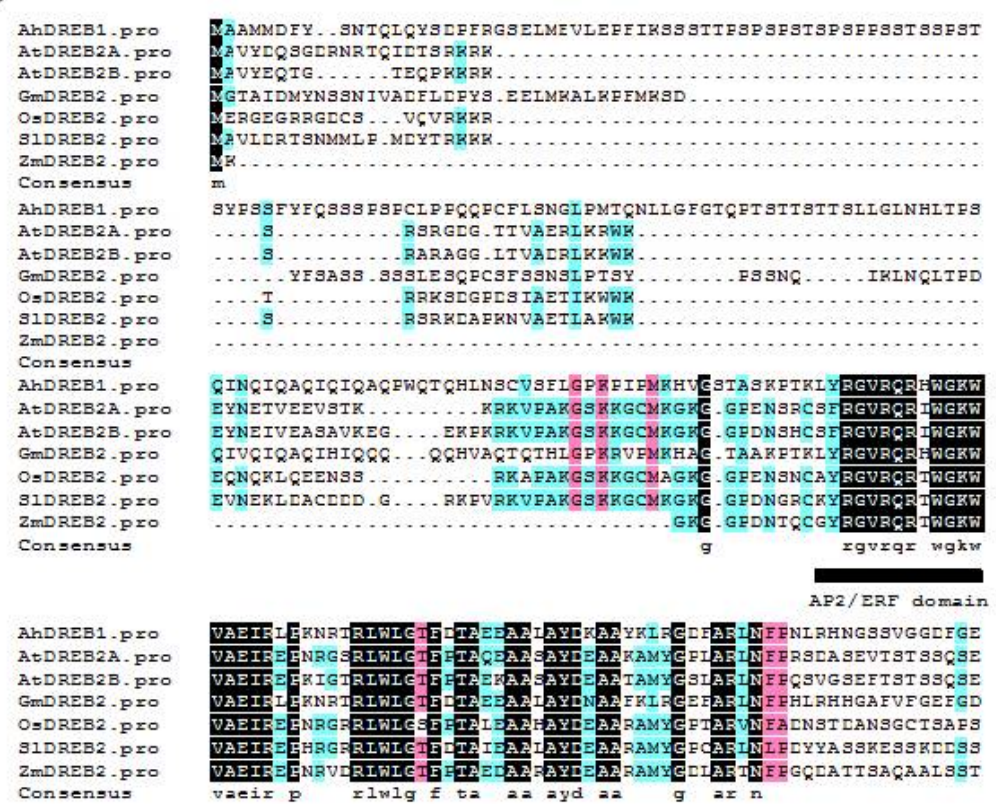

AhDREB1.PYO

AtDREB2A - pro ATDREB2B. PIO GmDREBR - P 20 OADREB - $P$ IO SIDREB - P Consensus

AhDREB1.P 00

AtDREB2A . P 0 AtDREB2B.PIO GMDREB2-P OSDREBL-PIO ZIDREB - P IO Consensus AhDREB1 pro AhDREB1.pro AtDREB2B - pro GmDREB2 - pro OSDREB 2 - P $=0$ SIDREB2 PXO ZMDREB 2 - P 10 Consensus AhDREB1.pro AtDREB2B , $p r$ GMDREB2.PXO OSDREB 2 pro SIDREB2 - P rO ZMDREB2 - $P$ ×O Consensus AhDREBI.pro AtDREa2A ADDEB OIDREB2 - P YO SIDREB2 PYO 2 MDREB2,pro ZMDREB2.pI

\begin{tabular}{|c|c|}
\hline$\ldots$ AGAAGSKAAVSKR & 286 \\
\hline VCTVET FGCVH . . . VRTEDEDCESMFFSG . . GVEFMYCIEN . . . . . GAEEMKRGVRAD & 198 \\
\hline VCIVENKAVVCGLVCVKHEDTDCESNFFSQIILVREESCGTRPDSCTVGHCLDMNSSINYD & 209 \\
\hline 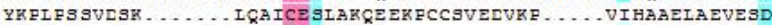 & 240 \\
\hline 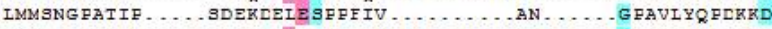 & 186 \\
\hline IFTVSRSISNT . . . ASSFBEVCPAGLMMRGRANVFAARHELRSIEIDGARIGSNEIGTP & 210 \\
\hline SACAAPTAVEA... IQTIGISCESTITSNHSIIAST BHKIE . . . . ASIISSYLKEKCP & 135 \\
\hline 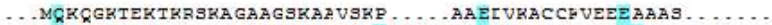 & 304 \\
\hline ..GVEPMYCIEN ...... GAEEMKRGVRADKHWISEEFEHNYWS -IIIRERER .. & 219 \\
\hline 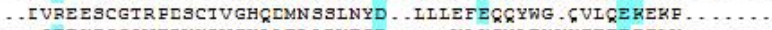 & 229 \\
\hline 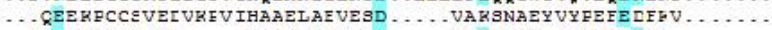 & 258 \\
\hline 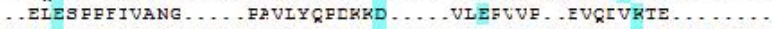 & 201 \\
\hline 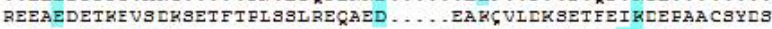 & 269 \\
\hline 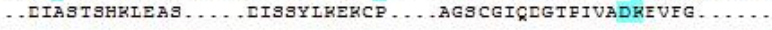 & 155 \\
\hline 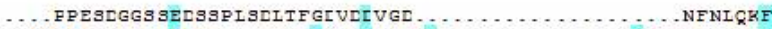 & \\
\hline 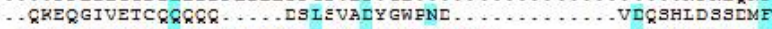 & 259 \\
\hline 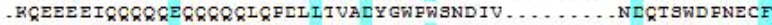 & 279 \\
\hline .... EHENEMFSGESSSPESEVTFIDFSDFSISNNCূW ..... & 302 \\
\hline$\ldots$ GSNGIREVCCRERKNMEVC.ESEGIVIHREVNISY $\ldots \ldots \ldots \ldots$ EYFNVHEVVEM & 245 \\
\hline 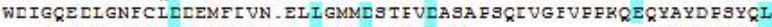 & 328 \\
\hline 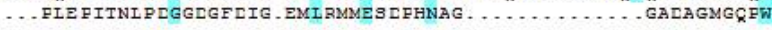 & 197 \\
\hline FSYEIDWLSI. . & 349 \\
\hline 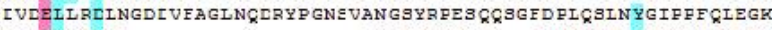 & 319 \\
\hline 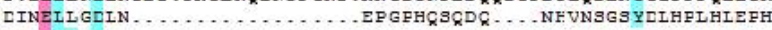 & 317 \\
\hline FEVEIDWEAI & 312 \\
\hline$\ldots \ldots \ldots \ldots$ TEVHEEY & 274 \\
\hline 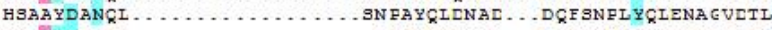 & 367 \\
\hline 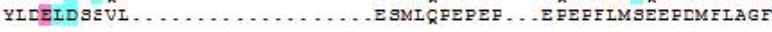 & 235 \\
\hline & 349 \\
\hline DGNGFFIIISYLIIEN & 335 \\
\hline IG_HEFNGISSIII $\ldots \ldots \ldots \ldots \ldots \ldots \ldots \ldots \ldots$ & 330 \\
\hline & 312 \\
\hline 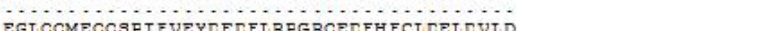 & 274 \\
\hline 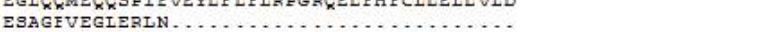 & 248 \\
\hline
\end{tabular}

Figure 1. Cont. 
(B)

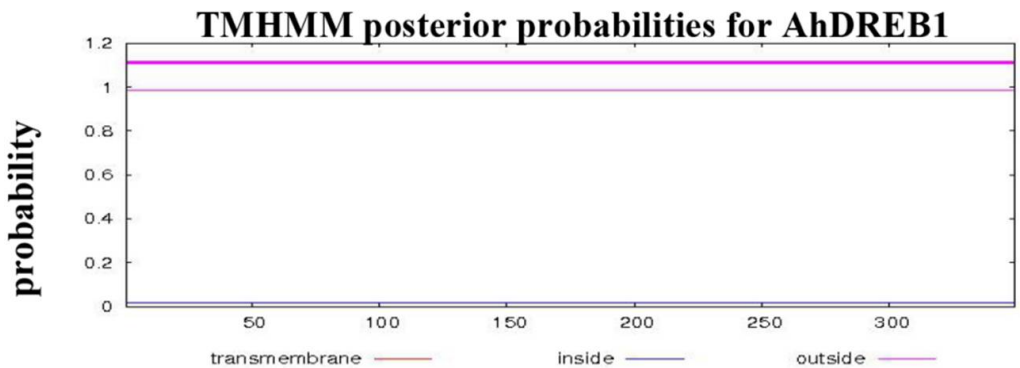

(C)

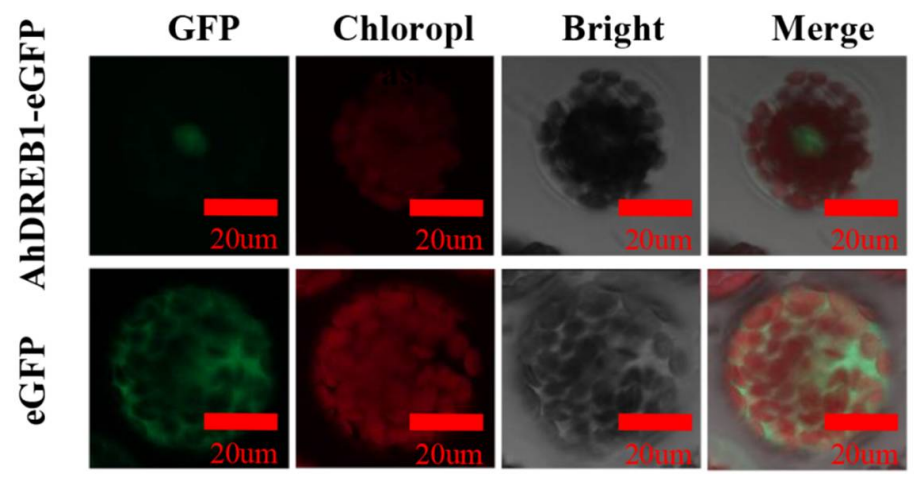

Figure 1. AhDREB1 is a member of the APETALA2/Ethylene Responsive Factor (AP2/ERF) transcription factor family in peanut. (A) Alignment of amino acid sequences of AhDREB1 with six dehydration-responsive element binding protein (DREB) subfamily proteins. AhDREB1: GenBank number API65088.1; AtDREB2A: GenBank number BAA33794.1; AtDREB2B: GenBank number BAA33795.1; GmDREB2: GenBank number AAQ57226.1; OsDREB2: GenBank number AAN02487.2; SIDREB2: GenBank number ADZ15315.1; ZmDREB2: GenBank number AFI71287.1. Identical amino acid residues are shaded in black. Conservative AP2/ERF domain are marked with a black underline. (B) AhDREB1 has no transmembrane domain, transmembrane structure was analyzed using online TMHMM 2.0 (http://www.cbs.dtu.dk/services/). (C) The intracellular localization of AhDREB1 in Arabidopsis protoplasts. Image showed the eGFP protein was observed in the whole Arabidopsis protoplasts cells, which the AhDREB1-eGFP fusion protein was localized in nucleus in Arabidopsis protoplasts cells, determined by confocal and bright-field microscopy.

\subsection{Expression of AhDREB1 Is Induced by Osmotic Stress and ABA Treatment}

The expression profile of AhDREB1 was examined in peanut leaves treated with 20\% PEG 6000 or $100 \mu \mathrm{M}$ ABA. AhDREB1 expression was increased at $2 \mathrm{~h}$ of PEG treatment, slightly declined at $5 \mathrm{~h}$, then significantly increased and maintained a relatively high level from 8 to $24 \mathrm{~h}$. The drought-related genes AhAREB1 and AhNCED1 were also expressed in response to PEG. AhAREB1 levels did not change significantly at time points up to $5 \mathrm{~h}$, but then were significantly upregulated at $8 \mathrm{~h}$, and continuously increased from 12 to $24 \mathrm{~h}$. The expression of AhNCED1 was significantly increased at $2 \mathrm{~h}$, slightly decreased at 5 to $8 \mathrm{~h}$, although it remained higher than the control level, then slowly increased from 12 to $24 \mathrm{~h}$ (Figure 2). The expression of AhDREB1 could be induced by osmotic stress caused by PEG treatment suggests that AhDREB1 itself is a drought-responsive gene. Consistent with this, we found that $100 \mu \mathrm{M}$ ABA treatment produced a remarkable increase in AhDREB1 expression level at 2 to $5 \mathrm{~h}$, which slightly declined at $8 \mathrm{~h}$, then significantly increased at $12 \mathrm{~h}$, and finally declined again at $24 \mathrm{~h}$. In comparison, AhAREB1 expression continuously increased from 2 to $12 \mathrm{~h}$, and then declined $24 \mathrm{~h}$ after ABA treatment (Figure 3). Thus, AhDREB1 expression can be increased by both osmotic stress and treatment with exogenous ABA. 


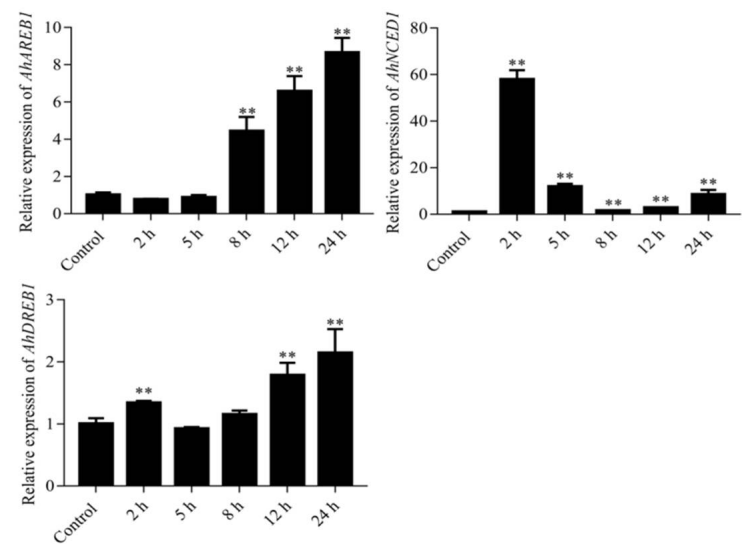

Figure 2. Expression analyses of AhDREB1, AhNCED1 and AhAREB1 following 20\% PEG treatment in peanut leaves by real-time quantitative PCR (RT-qPCR). Time points of 2, 5, 8, 12, and $24 \mathrm{~h}$ were sampled to observe the expression changing trend. The untreated group was used as the control. Mean and SD were obtained from more than three biological replicates. Asterisks indicate significant differences from control (Student's $t$ test $p$ values, ${ }^{* *} p<0.01$ ).

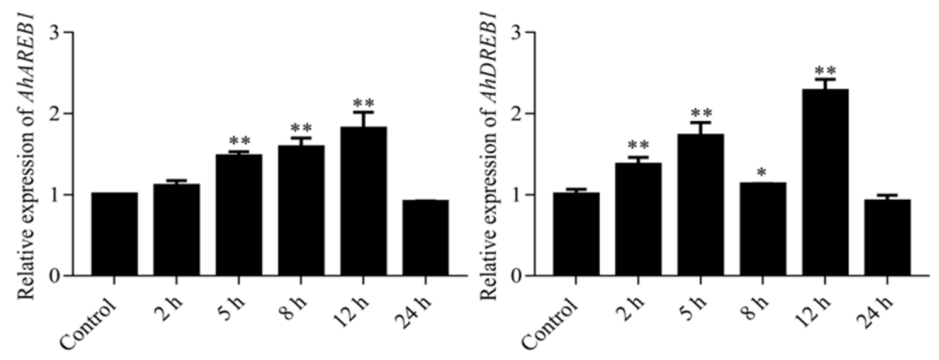

Figure 3. Expression analyses of AhDREB1 and AhAREB1 following $100 \mu \mathrm{M}$ abscisic acid (ABA) treatment in peanut leaves by RT-qPCR. Time points of 2, 5, 8, 12, and $24 \mathrm{~h}$ were sampled to observe the expression changing trend. The untreated group was used as the control. Mean and SD were obtained from more than three biological replicates. Asterisks indicate significant differences from control (Student's $t$ test $p$ values, ${ }^{*} p<0.05$ and ${ }^{* *} p<0.01$ ).

\subsection{Overexpression of AhDREB1 in Arabidopsis Enhances Plant Tolerance to Drought Stress}

AhDREB1 overexpression (AhDREB1-OX) Arabidopsis lines were obtained to understand the role of $A h D R E B 1$ in the drought stress response. Of more than 20 independent lines produced, three independent $\mathrm{T}_{3}$ homozygous $A h D R E B 1-O X$ lines $(O X-1, O X-2, O X-3)$ with a relatively high level of $A h D R E B 1$ transcription were selected for further studies (Figure $4 \mathrm{~A}$ ). We first examined whether $A h D R E B 1-O X$ lines at the seedling stage display tolerant phenotypes under drought stress conditions. When three-week-old Col and AhDREB1-OX lines were drought stressed for two weeks, OX-2 and $O X-3$ plants exhibited strong drought tolerance, while Col and $O X-1$ plants showed severe wilting (Figure 4C). After $2 \mathrm{~d}$ rehydration, almost all wild-type and $O X-1$ plants failed to recover, while $O X-2$ and $O X-3$ plants recovered rapidly (Figure $4 \mathrm{C}$ ). After recovery, $68 \%$ of $O X-2$ and $77 \%$ of $O X-3$ plants survived, in contrast to only $30 \%$ of $\mathrm{Col}$ and $24 \%$ of $O X-1$ plants (Figure $4 \mathrm{~B}$ ). We found that water content in AhDREB1-OX lines was not significantly different to that of Col plants during dehydration treatment (Figure 4D), but the stomatal aperture in leaves of OX-2 plants was smaller than in Col plants under stress conditions (Figure 4E) (Supplementary Figure S3). The endogenous ABA levels in the rosette leaves of all three independent $T_{3}$ homozygous transgenic lines were significantly higher than $\mathrm{Col}$ under normal growth conditions. Intriguingly, however, ABA content of $O X-2$ and $O X-3$ plants was higher than that in Col plants under drought stress conditions, although $O X-1$ ABA levels were not different to controls (Figure 4F). 

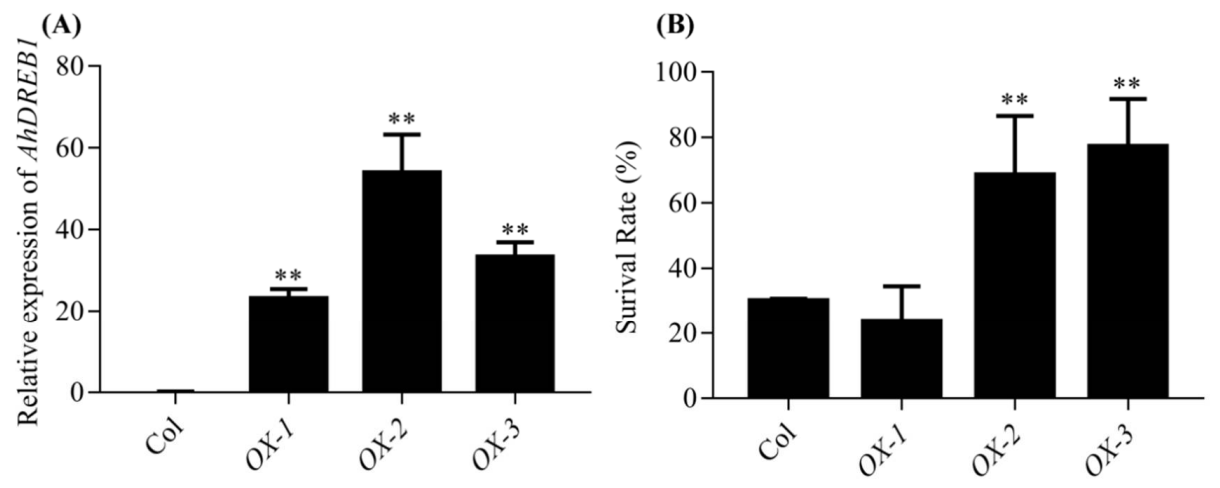

(C)
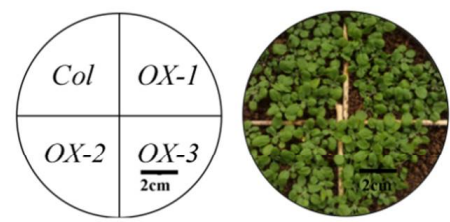

Untreated

(D)
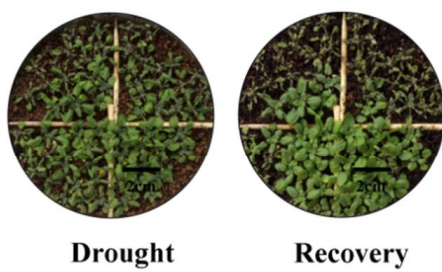

(E)
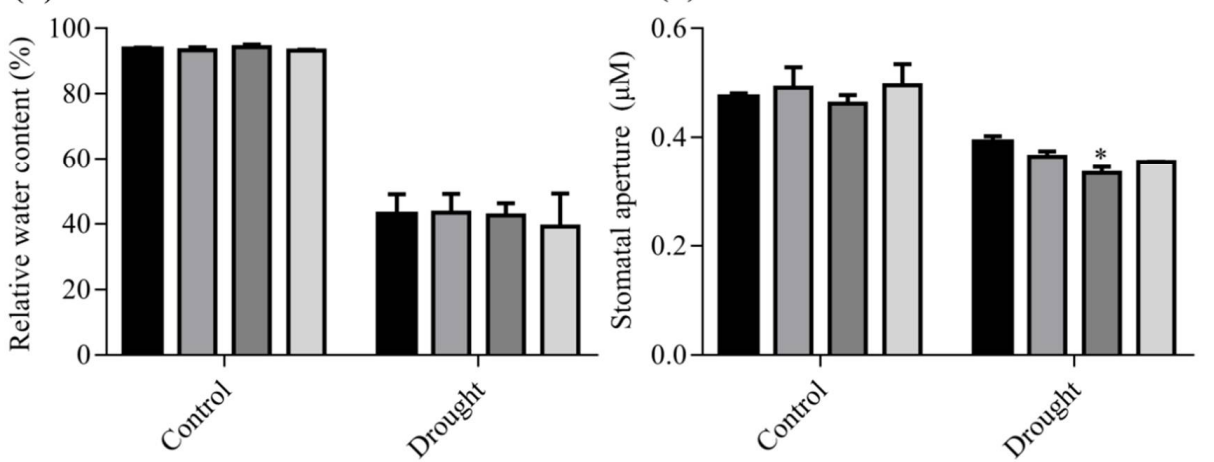

(F)

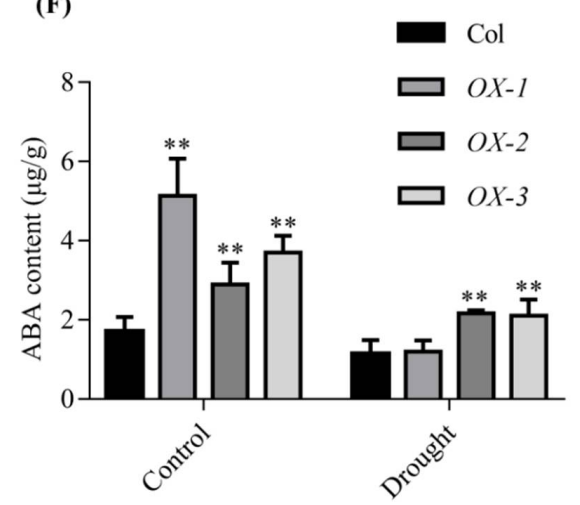

Figure 4. Overexpression of $A h D R E B 1$ in Arabidopsis enhances plant tolerance to drought stress. (A) The expression levels of AhDREB1 in AhDREB1-OX lines. (B) Survival rate of 3-week-old wild-type and AhDREB1-OX lines during the drought stress test. (C) Drought tolerance phenotype of 3-week-old wild-type and AhDREB1-OX lines were drought stress for 2 weeks and rehydration 2days. (D) The change in water content in overexpression Arabidopsis thaliana. (E) Stomatal opening in the leaves of 3-week-old wild-type and AhDREB1-OX lines under control conditions or after drought stress for 2 weeks; $n=180$. ' ${ }^{* \prime}$ indicates a significant difference at the level of $p<0.05$ between AhDREB1-OX lines and $\mathrm{Col}$ plants under control treatment or drought stress conditions. (F) ABA content in the leaves of 3-week-old wild-type and AhDREB1-OX lines under control conditions or after drought stress for 2 weeks. All experiments, mean and SD were obtained from more than three biological replicates. Asterisks in (A) to (F), indicate significant differences from Col (Student's $t$ test $P$ values, ${ }^{*} p<0.05$ and ** $p<0.01)$. 
Given that AhDREB1-OX plants showed enhanced drought tolerance, we investigated whether the transcription of drought stress-related marker genes was altered compared to Col control plants. We found that transcript levels of the drought stress-related genes AtRD29A, AtNCED3, AtP5CS1 and AtP5CS2 were significantly higher than Col in all three transgenic lines after drought treatment, especially in OX-2 plants (Figure 5). At the same time, the transcriptional level of AhDREB1 was also significantly increased during PEG treatment. These results indicate that overexpression of AhDREB1 can promote downstream drought-related gene expression. In summary, overexpression of AhDREB1 can enhance drought resistance of the vegetative stages of $A$. thaliana by increasing ABA levels and promoting the expression of drought-resistance genes.
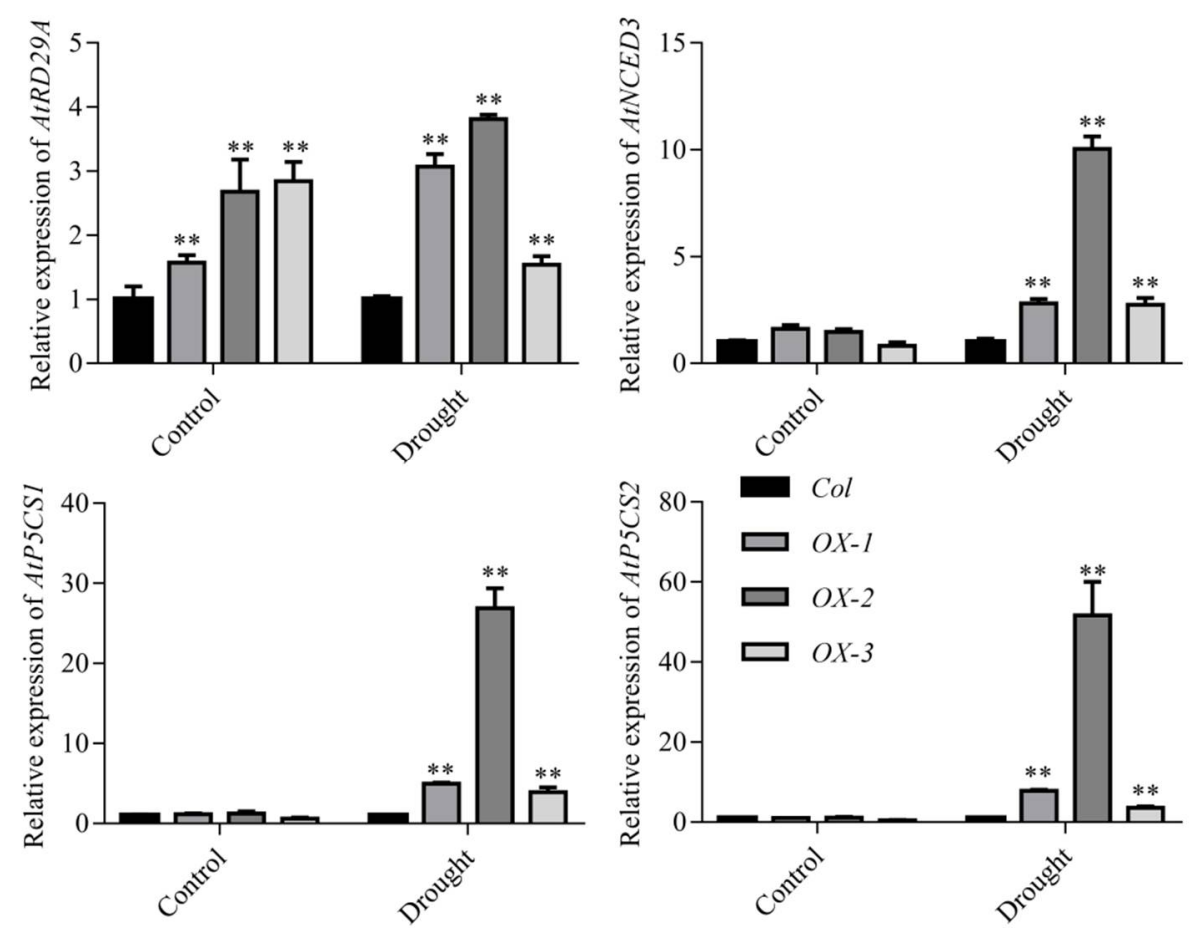

Figure 5. Expression analyses of drought stress-related marker genes: AtRD29A, AtNCED3, AtP5CS1, AtP5CS2 in the wild type and transgenic plants under drought stress. The untreated group was used as the control. Mean and SD were obtained from more than three biological replicates. Asterisks indicate significant differences from control (Student's $t$ test $P$ values, ${ }^{* *} p<0.01$ ).

\subsection{AhDREB1 Increases ABA Sensitivity and Affects ABA Signaling Pathway in AhDREB1-OX Arabidopsis Plants}

To investigate whether AhDREB1 affects plant drought tolerance via the ABA-dependent pathway, we checked ABA sensitivity of AhDREB1-OX transgenic plants at the germination stage. Seeds of the three overexpression lines, together with Col controls, were germinated in one-half-strength Murashige and Skoog $(1 / 2 \mathrm{MS})$ medium containing ABA at one of three different concentrations $(0,0.5,2 \mu \mathrm{M})$. AhDREB1-OX seeds exhibited a significantly slower germination rate than wild type following 0.5 and $2 \mu \mathrm{M}$ ABA treatment, but there were no differences between AhDREB1-OX and Col plants without ABA treatment (Figure 6A-D). Thus, ABA sensitivity of AhDREB1-OX plants was increased. Furthermore, the transcription levels of ABA signaling pathway-related genes AtPYL2, AtPP2C5, AtSnRK2.2, AtSnRK2.4, AtAREB3 and AtABF4 were significantly higher than in Col plants under both normal growth conditions and after exogenous application of $10 \mu \mathrm{M}$ ABA (Figure 7). In conclusion, AhDREB1 increases ABA sensitivity and affects the ABA signaling pathway in AhDREB1-OX Arabidopsis plants. 


\subsection{Histone Acetylation Is Involved in AhDREB1 Transcriptional Regulation}

Trichostatin A (TSA) is a small-molecule histone deacetylase (HDAC) inhibitor, that is, it represses histone deacetylation. We found that TSA treatment of peanut leaves slightly reduced the expression of $A h D R E B 1$ at 2 to $5 \mathrm{~h}$, while a relatively high transcription level of $A h D R E B 1$ was detected at 8 to $12 \mathrm{~h}$, followed by a decrease in expression at $24 \mathrm{~h}$ (Figure $8 \mathrm{~A}$ ). When we checked the drought-related genes $A h A R E B 1$ and $A h N C E D 1$, we observed that their expression profiles were both essentially consistent with that of AhDREB1 during TSA treatment (Figure 8B,C). These results suggest that TSA can promote the expression of $A h D R E B 1$ and, therefore, that histone acetylation modification may be involved in its transcriptional regulation. To clarify this, chromatin immunoprecipitation (ChIP) assays were performed using antibodies that specifically recognize histone $\mathrm{H} 3 \mathrm{ac}$ modifications. H3ac levels in chromatin of peanut leaves treated with TSA and PEG were analyzed in various regions of the AhDREB1 gene using ChIP-quantitative PCR (qPCR). TSA treatment resulted in relatively high H3ac enrichment levels in the P4 and P5 regions of AhDREB1 chromatin, whereas no statistically significant changes in H3ac were detected in other regions (Figure 8E). Treatment with 20\% PEG also enriched levels of H3ac, but only in the P3, P5, P6 regions (Figure 8F). In contrast, the application of $100 \mu \mathrm{M}$ ABA (Supplementary Figure S4) produced no significant changes in H3ac levels in any region of AhDREB1 chromatin. In conclusion, histone acetylation is involved in $A h D R E B 1$ transcriptional regulation and the level of the histone acetylation in AhDREB1 chromatin can be modulated by both TSA and $20 \%$ PEG treatment.

(A)

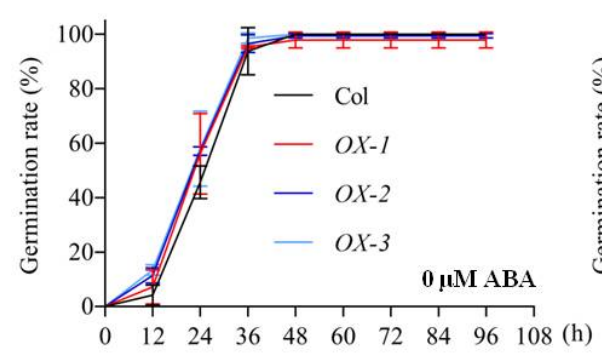

(C)

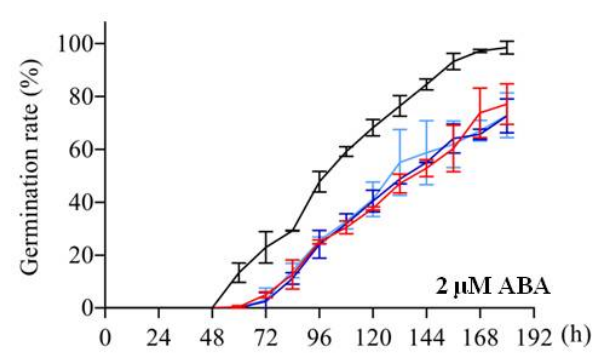

(B)

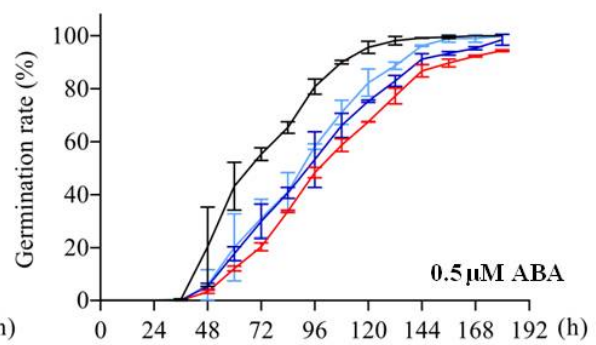

(D)

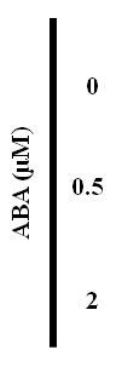

Col $\quad O X-1 \quad O X-2 \quad O X-3$

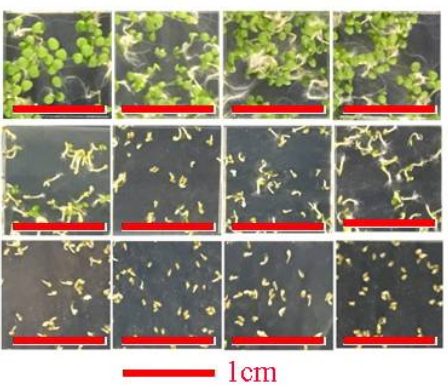

Figure 6. AhDREB1 increases ABA sensitivity in Arabidopsis. (A-C) Seed germination rate of AhDREB1-OX lines and Col in response to different concentrations of ABA. Numbers of germinated seedlings were recorded from 0 to $192 \mathrm{~h}$ after stratification on one-half-strength Murashige and Skoog (1/2 MS) agar plates containing 0, 0.5, or $2 \mu \mathrm{M}$ ABA, respectively. (D) Photographs of seed germination on agar plates containing $0,0.5$ or $2 \mu \mathrm{M}$ ABA. Mean and SD were obtained from more than three biological replicates. 

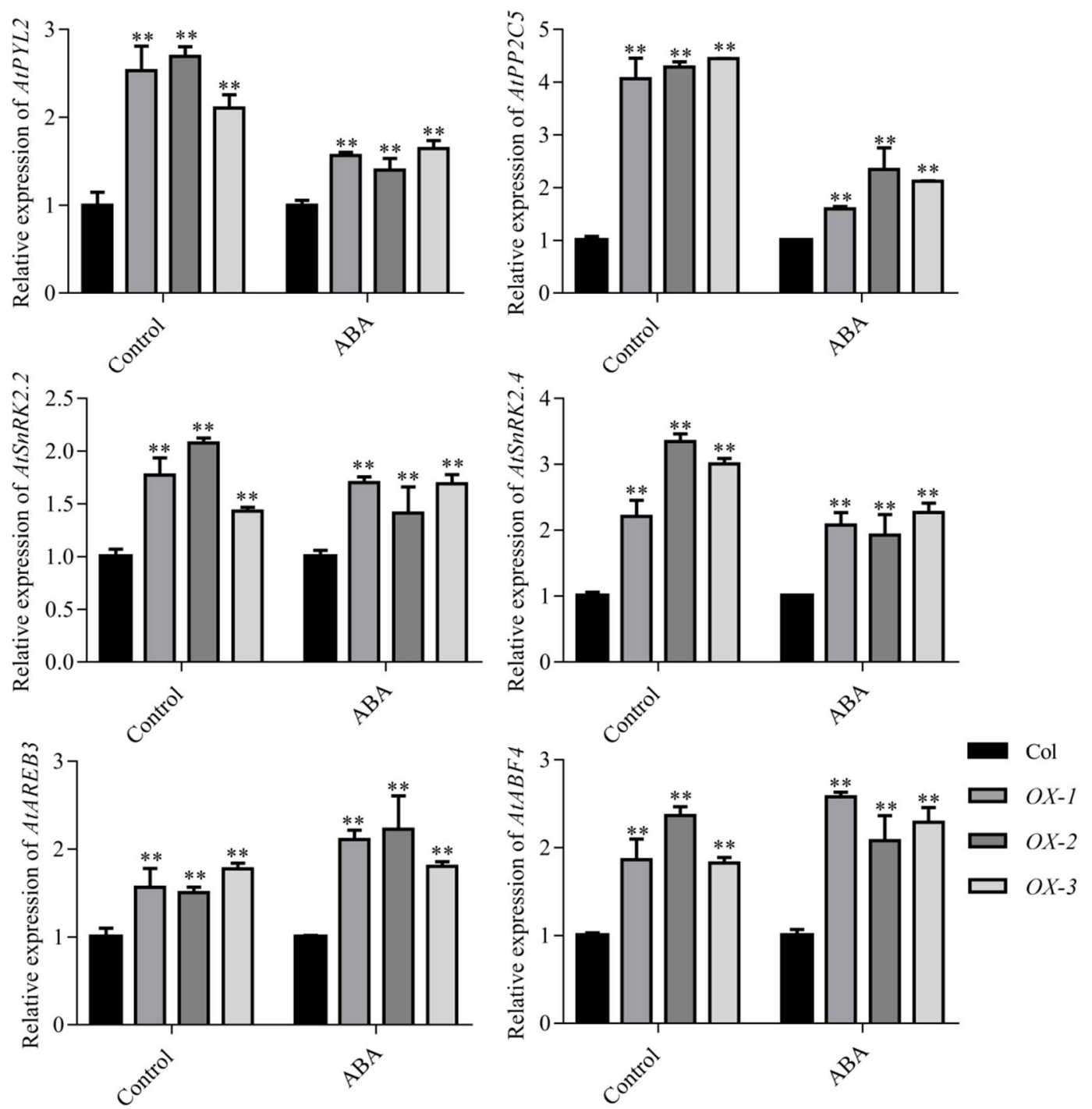

Figure 7. Expression changes of ABA signaling pathway related gene: AtPYL2, AtPP2C5, AtSnRK2.2, AtSnRK2.4, AtAREB3, AtABF4 following $10 \mu \mathrm{M}$ ABA treatment. The untreated group was used as the control. Mean and SD were obtained from more than three biological replicates. Asterisks indicate significant differences from control (Student's $t$ test $p$ values, ${ }^{* *} p<0.01$ ). 
(A)

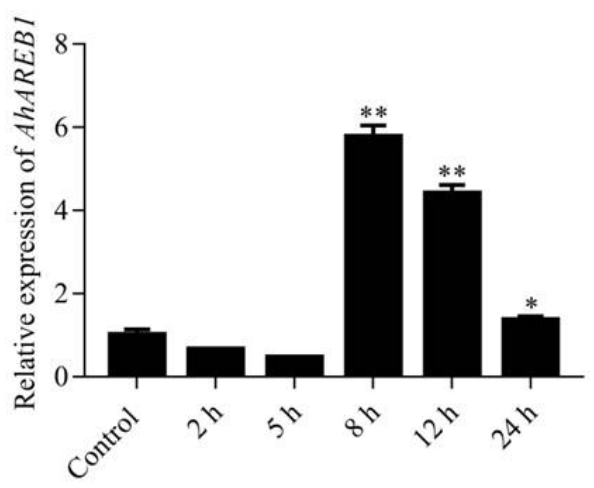

(C)

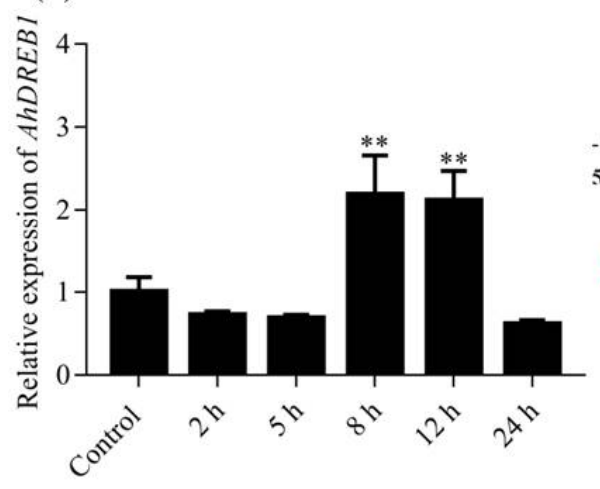

(E)

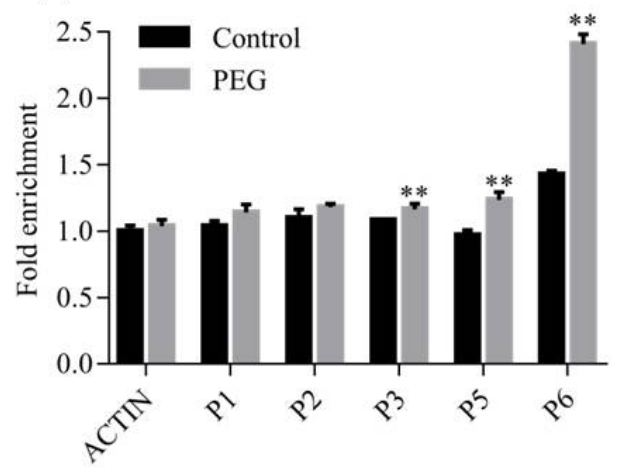

(B)

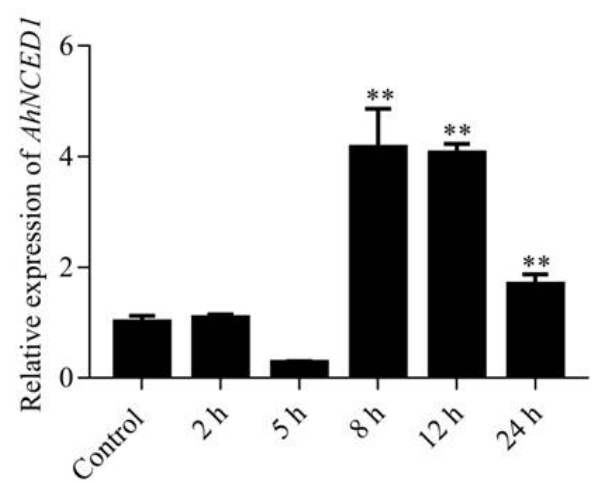

(D)

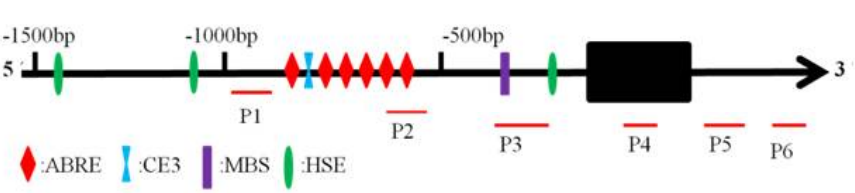

(F)

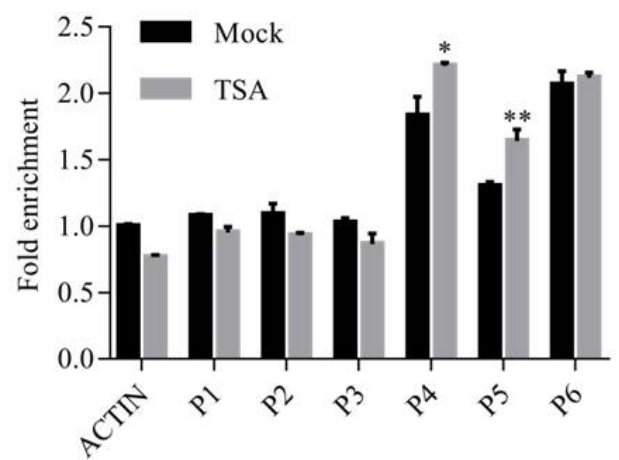

Figure 8. Histone acetylation is involved in AhDREB1 transcriptional regulation. (A-C) Expression analyses of $A h D R E B 1$ and stress resistance genes following $1 \mu \mathrm{M}$ treatment in peanut leaves by RT-qPCR. Time points of 2, 5, 8, 12, and $24 \mathrm{~h}$ were sampled to observe the expression changing trend. The untreated group was used as the control. Mean and SD were obtained from more than three biological replicates. Asterisks indicate significant differences from control (Student's $t$ test $p$ values, $* p<0.05$ and ${ }^{* *} p<0.01$ ). (D) The structure of the AhDREB1 promoter. PCR amplification (P1-P6) for chromatin immunoprecipitation (ChIP) assays are indicated. (E,F) H3ac levels in chromatin of peanut leaves under $1 \mu \mathrm{M}$ TSA and 20\% PEG treatment, respectively. The untreated group was used as the control. Mean and SD were obtained from more than three biological replicates. Asterisks indicate significant differences from control (Student's $t$ test $p$ values, ${ }^{*} p<0.05$ and ${ }^{* *} p<0.01$ ).

\section{Discussion}

$\mathrm{AP} 2 / \mathrm{ERF}$ are plant-specific transcription factors. Increasing evidence shows that AP2/ERF TF genes are induced in response to various stresses, including drought, abscisic acid, salt, cold, heat and alkalinity [42]. Here, we isolated full-length CDS of AhDREB1 gene, which encodes an 
AP2/ERF TF of the DREB subfamily (Figure 1A). The expression of AhDREB1 is induced by PEG 6000 and ABA (Figures 2 and 3), which suggests that AhDREB1 might play an important role in the plant drought response and the ABA signaling pathway. A large number of studies have revealed that AP2/ERF TFs can enhance the stress tolerance when overexpressed in transgenic plants [20,43]. For instance, overexpression of $E R F 1-V$ in wheat improves resistance to powdery mildew, salt and drought stress [44]. Similarly, overexpression of SpERF1 enhances drought tolerance of transgenic A. thaliana [45]. It has been reported that overexpression of OsERF71 can enhance the drought stress tolerance of rice by changing the morphological structure of roots and by regulating the expression of genes related to lignin synthesis and drought-related genes [46]. In the current paper, overexpression of AhDREB1 significantly enhanced drought resistance in A. thaliana (Figure 4C) in the vegetative stages of at least two OX lines (Figure 4B).

It is well known that ABA is a pivotal regulator of plant responses to drought stress. The level of ABA increases rapidly under drought stress and this is a function of its biosynthesis, catabolism and transport [10]. NCED is a key enzyme in the ABA synthesis pathway $[10,11]$ and, in Arabidopsis, the respective gene $A t N C E D 3$, which is induced by drought stress, controls the level of endogenous ABA under drought-stress conditions. Overexpression of AtNCED3 causes an increase in endogenous ABA levels and promotes transcription of drought- and ABA-inducible genes, which improve drought tolerance in Arabidopsis [47]. In peanut plants, the expression of AhNCED1 is significantly upregulated by dehydration and high salinity, while overexpression of AhNCED1 in Arabidopsis plants leads to the accumulation of endogenous ABA and improved water-stress tolerance [48]. ABA transport is also an important factor affecting ABA levels and plant responses to drought stress. Previous studies have shown that mutation of the Arabidopsis abcg40 gene, which encodes an ABA transporter, causes stomata to close more slowly in response to $\mathrm{ABA}$, and strongly delays the upregulation of ABA-responsive genes, resulting in reduced drought tolerance [49]. In peanut, AhATL1, an ABCG transporter subfamily gene, is upregulated by water stress and exogenous ABA treatment. Overexpression of AhATL1 decreases ABA sensitivity and drought resistance by influencing ABA transport in Arabidopsis [50]. These studies show that the level of ABA is closely related to drought tolerance and thus ABA levels can be used as an important index of drought resistance in plants [9]. Consistent with their drought-resistant phenotype, we showed here that ABA contents of the Arabidopsis AhDREB1-OX lines $O X-2$ and $O X-3$ were significantly higher than in Col under drought stress, although line OX-1 showed no significant difference to the control (Figure 4F). We suspected that the phenomena may be related to the expression of AhDREB1 in Arabidopsis thaliana. Indeed, AhDREB1 expression in OX-1 line is relatively low compared to the other two lines (Figure 4A), which may affect the level of ABA under drought stress and affect the drought resistance of Arabidopsis. It is interesting that the ABA levels of AhDREB1-OX lines were also significantly higher than Col under normal growth conditions. Previous studies have shown that ABA is an important factor in regulating seed dormancy and germination [51]. In Arabidopsis, strains overexpressing AtDREB2C are ABA hypersensitive during germination and show enhanced plant tolerance to cold and heat stress [8]. The overexpression of GhERF38 increased ABA sensitivity in seed germination and seedling period in Arabidopsis [52]. Our AhDREB1-OX lines were also ABA hypersensitive during seed germination and seedling development. Thus we speculated that AhDREB1 may affect ABA signaling pathway in Arabidopsis. To verify this hypothesis, we checked expression levels of ABA signaling pathway-related genes in AhDREB1-OX lines and Col plants subjected to $10 \mu \mathrm{M}$ ABA treatment. All of these genes showed significantly higher expression than the wild-type under both normal conditions and during ABA treatment (Figure 7). These results suggest AhDREB1 increases ABA sensitivity and affects ABA signaling pathway in Arabidopsis.

It has been reported that ERF and DREB subfamily TFs can directly interact with GCC boxes (GCCGCC) or DRE elements in the promoters of downstream stress- and defence-related genes to regulate multiple stress responses [20]. Overexpression of SpERF1 enhances drought tolerance of transgenic $A$. thaliana after binding to DRE elements in the promoters of the abiotic stress-responsive genes P5CS1, RD29A, HSP101, LEA4-5, HSP70 and COR47 [45]. ThCRF1 binds DRE and CGG-box 
motifs and induces the expression of various genes, including P5CS, SOD and $P O D$, thereby enhancing tolerance to salt stress [17]. In this paper, to further investigate the molecular mechanism by which $A h D R E B 1$ enhances drought tolerance, RT-qPCR was carried out to quantify transcription levels of RD29A, P5CS1, P5CS2 and NCED1. All four genes were significantly upregulated in AhDREB1-OX lines subjected to drought stress (Figure 5). This result was consistent with the drought-resistant phenotype described above. In summary, AhDREB1 affects ABA sensitivity and ABA signaling by increasing the level of endogenous $\mathrm{ABA}$ and the expression of downstream drought stress-related genes, which in turn increase $A$. thaliana drought resistance in overexpressing lines.

Histone acetylation is one of the most important histone modifications and can affect chromatin structure and gene expression. TSA, a small molecule HDAC inhibitor, can cause transient increases in acetylation of histone $\mathrm{H} 2 \mathrm{~B}, \mathrm{H} 4$, and $\mathrm{H} 3$ in chromatin [53]. It has been reported that $R A B 18, R D 29 B$ and $H S P 70$, together with four late embryogenesis abundant protein (LEA) genes, are upregulated by TSA during seed germination [54]. TSA also suppresses cold-induced transcription of the ZmDREB1 gene in maize [39]. In our studies, the expression of AhDREB1 was significantly upregulated by TSA (Figure $8 \mathrm{~A}$ ), implying that histone acetylation may be involved in the transcriptional regulation of AhDREB1. We investigated the effect of TSA treatment on the enrichment level of H3ac in AhDREB1 chromatin using ChIP-qPCR assays. The levels of H3ac were significantly increased in the P4 and P5 regions of AhDREB1 (Figure 8E), consistent with our AhDREB1 gene expression results following TSA treatment. We therefore conclude that histone acetylation is involved in the transcriptional regulation of AhDREB1.

Many studies have shown that histone acetylation plays an important role in the regulation of plant growth and development, and in the responses to abiotic stress. Thus, in Arabidopsis, H3K4me3 and H3K9ac levels increase in the coding regions of RD29A, RD29B, RD20 and RAP2.4 genes during drought stress [55]. HDACs positively affect the expression of the cold-induced ZmDREB1 gene, and this affects plant tolerance to cold stress [39]. Acetylation of H3K9 and K3K14 is increased under PEG and ABA treatment in A. hypogaea [36], while in A. thaliana, AtHDA9 negatively affects plant sensitivity to drought and salt stresses by modulating histone acetylation levels of a large number of stress-responsive genes [27]. In this paper, we found that overexpression of AhDREB1 enhances plant tolerance to drought stress and that AhDREB1 transcription is affected by histone acetylation. Accordingly, we hypothesised that AhDREB1 is affected by histone acetylation under drought conditions, and that this affects drought resistance in whole plants. To verify the hypothesis, we tested H3ac levels following treatment with 20\% PEG and found these to increase in the P3, P5, P6 regions of the AhDREB1 gene (Figure 8F). However, treatment with $100 \mu \mathrm{M}$ ABA yielded no significant change in H3ac levels (Supplementary Figure S4). In conclusion, histone acetylation is involved in the transcriptional regulation of AhDREB1 under osmotic stress conditions by increasing H3ac levels, thereby further improving plant drought resistance.

In summary, we isolated a full-length CDS of the AP2/ERF family gene AhDREB1 from peanut leaves and showed that AhDREB1 overexpression can improve the tolerance of Arabidopsis to drought via the ABA-dependent pathway. At the same time, histone acetylation is involved in the transcriptional regulation of $A h D R E B 1$ during osmotic stress, further improving drought resistance. This is the first report that histone acetylation is involved in the transcriptional regulation of a $D R E B$ subfamily gene in peanut. The findings provide new insight into how AP2/ERF TFs enhance plant tolerance to drought stress, which may lead to successful breeding of drought resistant strains of peanuts and other crops.

\section{Material and Methods}

\subsection{Peanut Plant Material and Growth Conditions}

Seeds of peanut (Arachis hypogaea L. cv. Yueyou 7) were provided by the Crop Research Institute, Guangdong Academy of Agricultural Sciences, China. Seeds were soaked in water for $12 \mathrm{~h}$, and then 
transferred to moist filter paper in an artificial climate incubator with a cycle of $16 \mathrm{~h}$ light from fluorescent and incandescent lamps $\left(200 \mu \mathrm{mol} \cdot \mathrm{m}^{-2} \cdot \mathrm{s}^{-1}\right)$ at $26{ }^{\circ} \mathrm{C}$ followed by $8 \mathrm{~h}$ darkness at $22{ }^{\circ} \mathrm{C}$ for $48 \mathrm{~h}$ until germination. Germinated seeds were planted in a potting mixture of vermiculite, perlite and soil (1:1:1) in the artificial climate incubator. Plants were watered with half-strength Murashige and Skoog nutrient solution every other day [36].

\subsection{Isolation and Sequence Analysis of AhDREB1 from Arachis hypogaea L.}

The total RNA of peanut leaves was extracted. Reverse transcription is performed by using 200 units Superscript III Reverse Transcriptase (Invitrogen, catalog no. 18080, Shanghai, China) and $500 \mathrm{ng}$ oligo-dT primer and then the first strand of cDNA was synthesized. Reverse transcribed (RT) conditions were: $70{ }^{\circ} \mathrm{C}$ for $10 \mathrm{~min}(\mathrm{~min}), 42^{\circ} \mathrm{C}$ for $1 \mathrm{~h}$, and $15 \mathrm{~min}$ at $70{ }^{\circ} \mathrm{C}$ [36]. The cDNA was used as a template to amplify AhDREB1 open reading frame (ORF) by PCR using the following primers: AhDREB1 ORF forward primer $5^{\prime}$-ATGGCAGCAATGATGGATTTCTACA-3', AhDREB1 ORF reverse primer $5^{\prime}$-TCACAGAGAATCCCAATCAATCTCA-3'. PCR amplification was performed as follows: $94{ }^{\circ} \mathrm{C}$ for $7 \mathrm{~min}$, then 35 cycles of $94{ }^{\circ} \mathrm{C}$ for $30 \mathrm{~s}(\mathrm{~s}), 55^{\circ} \mathrm{C}$ for $30 \mathrm{~s}$ and $72{ }^{\circ} \mathrm{C}$ for $1 \mathrm{~min} 30 \mathrm{~s}$, with a final extension step at $72{ }^{\circ} \mathrm{C}$ for $10 \mathrm{~min}$. PCR products were purified with an Agarose Gel DNA Purification Kit (TaKaRa, catalog no. DV805A, Dalian, China) and were ligated into the pMD19 T-vector (TaKaRa, catalog no. 6013, Dalian, China). Plasmids were sequenced on both strands.

Sequence analysis was performed using Lasergene 7.0 software (DNAStar, Madison, WI, USA). On-line BLAST analysis of DNA and amino acid sequences was performed at the National Center for Biotechnology Information Services website (https:/ / blast.ncbi.nlm.nih.gov/Blast.cgi). Multiple sequence alignment was performed using DNAMAN8.0 software [56]. The AhDREB1 promoter sequence was identified using the PlantCARE database (http: / / bioinformatics.psb.ugent.be/webtools / plantcare $/ \mathrm{html} /$ ). AhDREB1 was assessed for the presence of a transmembrane domain using online TMHMM 2.0 (http://www.cbs.dtu.dk/services/) [50]. Promoter elements were analyzed using PlantCARE [57,58].

\subsection{Subcellular Localization Analysis}

The expression vectors for subcellular localization were constructed as follows: the AhDREB1 coding region was prepared by PCR using the primer set forward primer 5'-ATGGCAGCAATGATGGATTTCTACA-3' and reverse primer 5'-TCACAGAGAATCCCAATCAAT CTCA-3'. The amplified fragment was cloned into pMD19 T-vector and sequenced on both strands. Subsequently, the AhDREB1 ORF was cloned into the p35S::eGFP vector [59]. The fusion construct p35S::AhDREB1-eGFP was driven by the 35S promoter. The recombinant plasmid vector was transformed into Arabidopsis protoplasts. After incubation for 12-18 h in the dark, eGFP fluorescence signals in protoplasts were observed under the confocal microscope (Carl Zeiss LSM 710; Carl Zeiss, Germany).

\subsection{Peanut Plant Treatments}

Peanut seedlings were removed from the soil at the four-leaf stage, and after the roots were carefully rinsed with deionized water, they were maintained in 1/8 MS medium for up to $2 \mathrm{~h}$. Seedlings were treated with $20 \%$ PEG or $100 \mu \mathrm{M}$ ABA for 2, 5, 8, 12 and $24 \mathrm{~h}$. Trichostatin A (TSA) was used to treat seedlings at $1 \mu \mathrm{M}$ for 2, 5, 8, 12 and $24 \mathrm{~h}$ [36]. Untreated seedlings planted in the soil were used as control plants. Seedlings placed in an equivalent volume of deionized water instead of ABA or TSA solutions were used as mock treatments. Seedlings were transferred to an artificial climate incubator $\left(26^{\circ} \mathrm{C}, 60 \%\right.$ moisture) under continuous light. Peanut leaves were harvested and frozen at $-80{ }^{\circ} \mathrm{C}$ prior to use. 


\subsection{Real-Time Quantitative PCR (RT-qPCR)}

Total RNA was extracted from peanut leaves or Arabidopsis thaliana seedlings [36]. For first-strand cDNA synthesis, $1 \mu \mathrm{g}$ high-quality total RNA was reverse transcribed (RT) using a Prime Script TM RT Reagent Kit (Perfect Real Time, TaKaRa, Dalian, China). The cDNA sample was used as the template for RT-qPCR. Relative transcript levels of AhDREB1 were evaluated in an ABI 7500 system using the ChamQ SYBR qPCR Master Mix (Low ROX Premixed, Vazyme Biotech Co., Ltd., Nanjing, China). Each reaction contained $5 \mu \mathrm{L} 2 \times$ ChamQ SYBR qPCR Master Mix, $10 \mathrm{ng}$ cDNA, $0.2 \mu \mathrm{L} 0.05 \mathrm{mM}$ stock solution of each primer in a final volume of $10 \mu \mathrm{L}$. The PCR thermal cycles were as follows: $95^{\circ} \mathrm{C}$ for $30 \mathrm{~s}$, followed by 40 cycles at $95^{\circ} \mathrm{C}$ for $5 \mathrm{~s}$ and $60^{\circ} \mathrm{C}$ for $34 \mathrm{~s}$. The AhACTIN and AtACTIN2 genes were used as internal controls for $A$. hypogaea and A. thaliana, respectively, as described [36,59]. Relative expression levels were calculated using the relative $2^{-\Delta \Delta C t}$ method [60]. The relevant primers for the analysis of gene transcription levels are listed one by one in Supplementary Table S1.

\subsection{Plant Transformation and Growth Conditions}

The recombinant vector p35S::AhDREB1-eGFP was transformed into Agrobaterium tumefaciens strain EHA105. Positive A. tumefaciens colonies containing the vector were employed for Arabidopsis Col transformation by the floral dip method [59]. $\mathrm{T}_{0}$ generation seeds were harvested and sowed on $1 / 2$ MS medium containing $50 \mathrm{mg} / \mathrm{L}$ kanamycin for selection, with repeat screening until $\mathrm{T}_{3}$. Individual plants were genotyped and homozygous plants were selected by PCR. Subsequent experiments were perform using $\mathrm{T}_{3}$ seeds. Arabidopsis growing conditions: light (a daily cycle of $16 \mathrm{~h}$ light and $8 \mathrm{~h}$ dark), temperature $\left(22 \pm 2{ }^{\circ} \mathrm{C}\right)$, relative humidity $(60-70 \%)$.

\subsection{Drought Stress Treatment of Arabidopsis}

The seeds of AhDREB1 overexpression lines and wild type Arabidopsis were grown in 1/2 MS medium and were vernalized for $48 \mathrm{~h}$ at $4{ }^{\circ} \mathrm{C}$. Then seeds were placed in a greenhouse with a daily cycle of $16 \mathrm{~h}$ light and $8 \mathrm{~h}$ dark at $20 \pm 2{ }^{\circ} \mathrm{C}$. After germination, the seedlings were planted in peat soil and grown for three weeks under the same light and temperature conditions. Three-week-old Arabidopsis thaliana was treated by soil drought for approximately 2 weeks under the same greenhouse conditions as above. The survival rate of Arabidopsis thaliana was counted after 2 days of rehydration. The survival rate was determined in three independent experiments [60].

\subsection{Stomatal Movement Assay}

To observe the stomatal opening of wild-type A. thaliana and AhDREB1-OX lines under normal growth and soil drought conditions, rosette leaves were detached from three-week-old plants. The detached rosette leaves were soaked in open stomatal buffer $\left(10 \mathrm{mM} \mathrm{MES}, 5 \mathrm{mM} \mathrm{KCl}, 50 \mathrm{mM} \mathrm{CaCl}_{2}\right.$, $\mathrm{pH}$ 6.15) in a growth chamber under incandescent lamps $\left(200 \mu \mathrm{mol} \cdot \mathrm{m}^{-2} \cdot \mathrm{s}^{-1}\right)$ at $20^{\circ} \mathrm{C}$ for $3 \mathrm{~h}$. Stomatal apertures were measured as described previously [49]. Digimizer software was used to measure stomatal apertures.

\subsection{Relative Water Content Determination}

Arabidopsis rosette leaves under normal growth and drought stress conditions were cut and collected to determine the fresh weight $\left(m_{f}\right)$. Leaf material was placed at $105{ }^{\circ} \mathrm{C}$ for $0.5 \mathrm{~h}$, then dried to constant weight at $80{ }^{\circ} \mathrm{C}$, giving the dry weight $\left(m_{d}\right)$. Water content $(\%)=\left(m_{f}-m_{d}\right) / m_{f} \times 100 \%$. The relative water content was determined in triplicate for each sample [61].

\subsection{Determination of $A B A$ Content}

As previously described, ABA was extracted from Arabidopsis rosette leaves under soil drought or control conditions [48]. Extraction in $80 \%(v / v)$ aqueous methanol, and high performance liquid chromatography fractionation in a SinoChrom ODS AP C18 column $(250 \times 4.6 \mathrm{~mm}, 5 \mu \mathrm{m}$, Dalian, 
China), were conducted as reported previously [62]. The level of ABA was determined in triplicate for each sample.

\subsection{ABA Sensitivity}

Sensitivity of AhDREB1-OX and wild-type seeds to ABA during seeds germination was assessed. To test the effects of $\mathrm{ABA}$ on germination, the seeds ( 80 seeds each, three repeats) were germinated on $1 / 2 \mathrm{MS}$ medium with $0.8 \%$ agar containing a gradient concentration of $\mathrm{ABA}(0,0.5,2 \mu \mathrm{M})$, and the germination rate of the treated seeds was calculated every $12 \mathrm{~h}$ until $192 \mathrm{~h}$ [59].

\subsection{ChIP-Quantitative PCR}

For the ChIP assay, leaves (500 mg) of four-leaf-stage (two weeks after planting) peanut plants were fixed with cold MC buffer (10 mM potassium phosphate $\mathrm{pH} 7.0,50 \mathrm{mM} \mathrm{NaCl}, 0.1 \mathrm{M}$ sucrose, $1 \%$ formaldehyde) for $20 \mathrm{~min}$ by vacuum concentration. Then add glycine to make its final concentration of $125 \mathrm{mM}$, vacuum incubation $10 \mathrm{~min}$ to terminate cross-linking. After washing leaves in $\mathrm{MC}$ buffer, leaves were collected and frozen in liquid nitrogen, and stored at $-80{ }^{\circ} \mathrm{C}$. The $\mathrm{ChIP}$ assay was performed as described previously [63]. One microgram of anti-H3ac and rabbit IgG (Millipore, Shanghai, China) were used for immunoprecipitation. Specifically, precipitated DNA was recovered and analyzed by ChIP-qPCR using SYBR Premix ExTaq Mix (Takara Bio, Dalian, China). Peanut ACTIN genes were used to calculate the relative fold-enrichment of target DNA fragments. The ChIP-qPCR primers are listed in Supplemental Table S2.

\subsection{Statistical Analysis}

Quantitative data were expressed as mean \pm SD. The statistical significance of experimental data was assessed by Student $t$-test or ANOVA (one-way analysis of variance with a Least Significant Difference (LSD) post-hoc test), as appropriate, using the SPSS17.0 statistical package (Chicago, IL, USA).

Supplementary Materials: Supplementary materials can be found at http:/ www.mdpi.com/1422-0067/19/5/1441/ s1.

Author Contributions: B.Z., L.S. and L.L. designed the research. B.Z. performed the research. B.Z., L.S. and B.H. analyzed the data and prepared figures. B.Z. wrote the manuscript in consulation with L.S. and L.L. L.L. and B.H. contributed reagents/materials/analysis tools.

Acknowledgments: We are grateful to Alan Tunnacliffe (Chief Editor) at Cambridge Academic Manuscripts, for technical support and critical reading of the manuscript. This work was supported by Natural Science Foundation of Guangdong Province (8151063101000011 to Ling Li), Innovation Project of Graduate School of South China Normal University (2017LKXM060 to Baihong Zhang).

Conflicts of Interest: The authors declare that the research was conducted in the absence of any commercial or financial relationships that could be construed as a potential conflict of interest.

\section{References}

1. Agarwal, P.K.; Gupta, K.; Lopato, S.; Agarwal, P. Dehydration responsive element binding transcription factors and their applications for the engineering of stress tolerance. J. Exp. Bot. 2017, 68, 2135-2148. [CrossRef] [PubMed]

2. Vinocur, B.; Altman, A. Recent advances in engineering plant tolerance to abiotic stress: Achievements and limitations. Curr. Opin. Biotechnol. 2005, 16, 123-132. [CrossRef] [PubMed]

3. Gonzalez-Villagra, J.; Rodrigues-Salvador, A.; Nunes-Nesi, A.; Cohen, J.D.; Reyes-Diaz, M.M. Age-related mechanism and its relationship with secondary metabolism and abscisic acid in Aristotelia chilensis plants subjected to drought stress. Plant Physiol. Biochem. PPB 2018, 124, 136-145. [CrossRef] [PubMed]

4. Liu, J.; Wang, R.; Liu, W.; Zhang, H.; Guo, Y.; Wen, R. Genome-Wide Characterization of Heat-Shock Protein 70s from Chenopodium quinoa and Expression Analyses of Cqhsp70s in Response to Drought Stress. Genes 2018, 9, 35. [CrossRef] [PubMed] 
5. Bohnert, H.J.; Jensen, R.G. Strategies for engineering water-stress tolerance in plants. Trends Biotechnol. 1996, 14, 89-97. [CrossRef]

6. Wang, W.X.; Vinocur, B.; Altman, A. Plant responses to drought, salinity and extreme temperatures: Towards genetic engineering for stress tolerance. Planta 2003, 218, 1-14. [CrossRef] [PubMed]

7. Yamaguchi-Shinozaki, K.; Shinozaki, K. Gene regulatory network in drought and cold stress responses. Plant Cell Physiol. 2005, 46, S33.

8. Lee, S.J.; Kang, J.Y.; Park, H.J.; Kim, M.D.; Bae, M.S.; Choi, H.I.; Kim, S.Y. DREB2C interacts with ABF2, a bZIP protein regulating abscisic acid-responsive gene expression, and its overexpression affects abscisic acid sensitivity. Plant Physiol. 2010, 153, 716-727. [CrossRef] [PubMed]

9. Roychoudhury, A.; Paul, S.; Basu, S. Cross-talk between abscisic acid-dependent and abscisic acid-independent pathways during abiotic stress. Plant Cell Rep. 2013, 32, 985-1006. [CrossRef] [PubMed]

10. Finkelstein, R. Abscisic Acid synthesis and response. Arabidopsis Book 2013, 11, e0166. [CrossRef] [PubMed]

11. Maruyama, K.; Urano, K.; Yoshiwara, K.; Morishita, Y.; Sakurai, N.; Suzuki, H.; Kojima, M.; Sakakibara, H.; Shibata, D.; Saito, K.; et al. Integrated Analysis of the Effects of Cold and Dehydration on Rice Metabolites, Phytohormones, and Gene Transcripts. Plant Physiol. 2014, 164, 1759-1771. [CrossRef] [PubMed]

12. Nakashima, K.; Ito, Y.; Yamaguchi-Shinozaki, K. Transcriptional regulatory networks in response to abiotic stresses in Arabidopsis and grasses. Plant Physiol. 2009, 149, 88-95. [CrossRef] [PubMed]

13. Fujita, Y.; Fujita, M.; Shinozaki, K.; Yamaguchi-Shinozaki, K. ABA-mediated transcriptional regulation in response to osmotic stress in plants. J. Plant Res. 2011, 124, 509-525. [CrossRef] [PubMed]

14. Singh, D.; Laxmi, A. Transcriptional regulation of drought response: A tortuous network of transcriptional factors. Front. Plant Sci. 2015, 6, 895. [CrossRef] [PubMed]

15. Yamaguchi-Shinozaki, K.; Shinozaki, K. A novel cis-acting element in an Arabidopsis gene is involved in responsiveness to drought, low-temperature, or high-salt stress. Plant Cell 1994, 6, 251-264. [CrossRef] [PubMed]

16. Yamaguchi-Shinozaki, K.; Shinozaki, K. Organization of cis-acting regulatory elements in osmotic- and cold-stress-responsive promoters. Trends Plant Sci. 2005, 10, 88-94. [CrossRef] [PubMed]

17. Qin, L.; Wang, L.; Guo, Y.; Li, Y.; Umut, H.; Wang, Y. An ERF transcription factor from Tamarix hispida, ThCRF1, can adjust osmotic potential and reactive oxygen species scavenging capability to improve salt tolerance. Plant Sci. 2017, 265, 154-166. [CrossRef] [PubMed]

18. Sakuma, Y.; Liu, Q.; Dubouzet, J.G.; Abe, H.; Shinozaki, K.; Yamaguchi-Shinozaki, K. DNA-binding specificity of the ERF/AP2 domain of Arabidopsis DREBs, transcription factors involved in dehydration- and cold-inducible gene expression. Biochem. Biophys. Res. Commun. 2002, 290, 998-1009. [CrossRef] [PubMed]

19. Riechmann, J.L.; Heard, J.; Martin, G.; Reuber, L.; Jiang, C.; Keddie, J.; Adam, L.; Pineda, O.; Ratcliffe, O.J.; Samaha, R.R.; et al. Arabidopsis transcription factors: Genome-wide comparative analysis among eukaryotes. Science 2000, 290, 2105-2110. [CrossRef] [PubMed]

20. Phukan, U.J.; Jeena, G.S.; Tripathi, V.; Shukla, R.K. Regulation of Apetala2/Ethylene Response Factors in Plants. Front. Plant Sci. 2017, 8, 150. [CrossRef] [PubMed]

21. Ohme-Takagi, M.; Shinshi, H. Ethylene-inducible DNA binding proteins that interact with an ethylene-responsive element. Plant Cell 1995, 7, 173-182. [CrossRef] [PubMed]

22. Yu, Y.; Duan, X.; Ding, X.; Chen, C.; Zhu, D.; Yin, K.; Cao, L.; Song, X.; Zhu, P.; Li, Q.; et al. A novel AP2/ERF family transcription factor from Glycine soja, GsERF71, is a DNA binding protein that positively regulates alkaline stress tolerance in Arabidopsis. Plant Mol. Biol. 2017, 94, 509-530. [CrossRef] [PubMed]

23. Scarpeci, T.E.; Frea, V.S.; Zanor, M.I.; Valle, E.M. Overexpression of AtERF019 delays plant growth and senescence, and improves drought tolerance in Arabidopsis. J. Exp. Bot. 2017, 68, 673-685. [PubMed]

24. Gilmour, S.J.; Sebolt, A.M.; Salazar, M.P.; Everard, J.D.; Thomashow, M.F. Overexpression of the Arabidopsis CBF3 transcriptional activator mimics multiple biochemical changes associated with cold acclimation. Plant Physiol. 2000, 124, 1854-1865. [CrossRef] [PubMed]

25. Liang, Y.; Li, X.; Zhang, D.; Gao, B.; Yang, H.; Wang, Y.; Guan, K.; Wood, A.J. ScDREB8, a novel A-5 type of $D R E B$ gene in the desert moss Syntrichia caninervis, confers salt tolerance to Arabidopsis. Plant Physiol. Biochem. 2017, 120, 242-251. [CrossRef] [PubMed]

26. Chen, J.Q.; Meng, X.P.; Zhang, Y.; Xia, M.; Wang, X.P. Over-expression of OsDREB genes lead to enhanced drought tolerance in rice. Biotechnol. Lett. 2008, 30, 2191-2198. [CrossRef] [PubMed] 
27. Zheng, Y.; Ding, Y.; Sun, X.; Xie, S.; Wang, D.; Liu, X.; Su, L.; Wei, W.; Pan, L.; Zhou, D.X. Histone deacetylase HDA9 negatively regulates salt and drought stress responsiveness in Arabidopsis. J. Exp. Bot. 2016, 67, 1703-1713. [CrossRef] [PubMed]

28. Luo, M.; Liu, X.; Singh, P.; Cui, Y.; Zimmerli, L.; Wu, K. Chromatin modifications and remodeling in plant abiotic stress responses. Biochim. Biophys. Acta 2012, 1819, 129-136. [CrossRef] [PubMed]

29. Swindell, W.R.; Huebner, M.; Weber, A.P. Plastic and adaptive gene expression patterns associated with temperature stress in Arabidopsis thaliana. Heredity 2007, 99, 143-150. [CrossRef] [PubMed]

30. Liu, X.; Yang, S.; Zhao, M.; Luo, M.; Yu, C.W.; Chen, C.Y.; Tai, R.; Wu, K. Transcriptional repression by histone deacetylases in plants. Mol. Plant 2014, 7, 764-772. [CrossRef] [PubMed]

31. Courey, A.J.; Jia, S. Transcriptional repression: The long and the short of it. Genes Dev. 2001, 15, $2786-2796$. [PubMed]

32. Hu, Y.; Qin, F.; Huang, L.; Sun, Q.; Li, C.; Zhao, Y.; Zhou, D.X. Rice histone deacetylase genes display specific expression patterns and developmental functions. Biochem. Biophys. Res. Commun. 2009, 388, $266-271$. [CrossRef] [PubMed]

33. Varotto, S.; Locatelli, S.; Canova, S.; Pipal, A.; Motto, M.; Rossi, V. Expression profile and cellular localization of maize Rpd3-type histone deacetylases during plant development. Plant Physiol. 2003, 133, 606-617. [CrossRef] [PubMed]

34. Yang, H.; Liu, X.; Xin, M.; Du, J.; Hu, Z.; Peng, H.; Rossi, V.; Sun, Q.; Ni, Z.; Yao, Y. Genome-Wide Mapping of Targets of Maize Histone Deacetylase HDA101 Reveals Its Function and Regulatory Mechanism during Seed Development. Plant Cell 2016, 28, 629-645. [CrossRef] [PubMed]

35. Luo, M.; Tai, R.; Yu, C.W.; Yang, S.; Chen, C.Y.; Lin, W.D.; Schmidt, W.; Wu, K. Regulation of flowering time by the histone deacetylase HDA5 in Arabidopsis. Plant J. 2015, 82, 925-936. [CrossRef] [PubMed]

36. Su, L.C.; Deng, B.; Liu, S.; Li, L.M.; Hu, B.; Zhong, Y.T.; Li, L. Isolation and characterization of an osmotic stress and ABA induced histone deacetylase in Arachis hygogaea. Front. Plant Sci. 2015, 6, 512. [CrossRef] [PubMed]

37. Luo, M.; Wang, Y.Y.; Liu, X.; Yang, S.; Lu, Q.; Cui, Y.; Wu, K. HD2C interacts with HDA6 and is involved in ABA and salt stress response in Arabidopsis. J. Exp. Bot. 2012, 63, 3297-3306. [CrossRef] [PubMed]

38. Mehdi, S.; Derkacheva, M.; Ramstrom, M.; Kralemann, L.; Bergquist, J.; Hennig, L. The WD40 Domain Protein MSI1 Functions in a Histone Deacetylase Complex to Fine-Tune Abscisic Acid Signaling. Plant Cell 2016, 28, 42-54. [PubMed]

39. Hu, Y.; Zhang, L.; Zhao, L.; Li, J.; He, S.; Zhou, K.; Yang, F.; Huang, M.; Jiang, L.; Li, L. Trichostatin A selectively suppresses the cold-induced transcription of the ZmDREB1 gene in maize. PLoS ONE 2011, 6, e22132. [CrossRef] [PubMed]

40. Zhao, X.; Li, C.; Wan, S.; Zhang, T.; Yan, C.; Shan, S. Transcriptomic analysis and discovery of genes in the response of Arachis hypogaea to drought stress. Mol. Biol. Rep. 2018, 45, 119-131. [CrossRef] [PubMed]

41. Li, X.Y.; Lu, J.B.; Liu, S.; Liu, X.; Lin, Y.Y.; Li, L. Identification of rapidly induced genes in the response of peanut (Arachis hypogaea) to water deficit and abscisic acid. BMC Biotechnol. 2014, 14, 58. [CrossRef] [PubMed]

42. Rong, W.; Qi, L.; Wang, A.; Ye, X.; Du, L.; Liang, H.; Xin, Z.; Zhang, Z. The ERF transcription factor TaERF3 promotes tolerance to salt and drought stresses in wheat. Plant Biotechnol. J. 2014, 12, 468-479. [CrossRef] [PubMed]

43. Mizoi, J.; Shinozaki, K.; Yamaguchi-Shinozaki, K. AP2/ERF family transcription factors in plant abiotic stress responses. Biochim. Biophys. Acta 2012, 1819, 86-96. [CrossRef] [PubMed]

44. Xing, L.; Di, Z.; Yang, W.; Liu, J.; Li, M.; Wang, X.; Cui, C.; Wang, X.; Wang, X.; Zhang, R. Overexpression of ERF1- $V$ from Haynaldia villosa Can Enhance the Resistance of Wheat to Powdery Mildew and Increase the Tolerance to Salt and Drought Stresses. Front. Plant Sci. 2017, 8, 1948. [CrossRef] [PubMed]

45. Yang, Y.; Dong, C.; Li, X.; Du, J.; Qian, M.; Sun, X.; Yang, Y. A novel AP2/ERF transcription factor from Stipa purpurea leads to enhanced drought tolerance in Arabidopsis thaliana. Plant Cell Rep. 2016, 35, 2227-2239. [CrossRef] [PubMed]

46. Lee, D.K.; Jung, H.; Jang, G.; Jeong, J.S.; Kim, Y.S.; Ha, S.H.; Do Choi, Y.; Kim, J.K. Overexpression of the OsERF71 Transcription Factor Alters Rice Root Structure and Drought Resistance. Plant Physiol. 2016, 172, 575-588. [CrossRef] [PubMed] 
47. Iuchi, S.; Kobayashi, M.; Taji, T.; Naramoto, M.; Seki, M.; Kato, T.; Tabata, S.; Kakubari, Y.; Yamaguchi-Shinozaki, K.; Shinozaki, K.; et al. Regulation of drought tolerance by gene manipulation of 9-cis-epoxycarotenoid dioxygenase, a key enzyme in abscisic acid biosynthesis in Arabidopsis. Plant J. 2001, 27, 325-333. [CrossRef] [PubMed]

48. Wan, X.R.; Li, L. Regulation of ABA level and water-stress tolerance of Arabidopsis by ectopic expression of a peanut 9-cis-epoxycarotenoid dioxygenase gene. Biochem. Biophys. Res. Commun. 2006, 347, 1030-1038. [CrossRef] [PubMed]

49. Kang, J.; Hwang, J.U.; Lee, M.; Kim, Y.Y.; Assmann, S.M.; Martinoia, E.; Lee, Y. PDR-type ABC transporter mediates cellular uptake of the phytohormone abscisic acid. Proc. Natl. Acad. Sci. USA 2010, 107, 2355-2360. [CrossRef] [PubMed]

50. Ge, K.; Liu, X.; Li, X.Y.; Hu, B.; Li, L. Isolation of an ABA Transporter-Like 1 Gene from Arachis hypogaea That Affects ABA Import and Reduces ABA Sensitivity in Arabidopsis. Front. Plant Sci. 2017, 8, 1150. [CrossRef] [PubMed]

51. Sah, S.K.; Reddy, K.R.; Li, J.X. Abscisic Acid and Abiotic Stress Tolerance in Crop Plants. Front. Plant Sci. 2016, 7, 571. [CrossRef] [PubMed]

52. Ma, L.F.; Hu, L.X.; Fan, J.B.; Amombo, E.; Khaldun, A.B.M.; Zheng, Y.; Chen, L. Cotton GhERF38 gene is involved in plant response to salt/drought and ABA. Ecotoxicology 2017, 26, 841-854. [CrossRef] [PubMed]

53. Waterborg, J.H. Plant histone acetylation: In the beginning ... . BBA-Gene Regul. Mech. 2011, 1809, 353-359. [CrossRef] [PubMed]

54. Tai, H.H.; Tai, G.C.C.; Beardmore, T. Dynamic histone acetylation of late embryonic genes during seed germination. Plant Mol. Biol. 2005, 59, 909-925. [CrossRef] [PubMed]

55. Kim, J.M.; To, T.K.; Ishida, J.; Morosawa, T.; Kawashima, M.; Matsui, A.; Toyoda, T.; Kimura, H.; Shinozaki, K.; Seki, M. Alterations of lysine modifications on the histone $\mathrm{H} 3 \mathrm{~N}$-tail under drought stress conditions in Arabidopsis thaliana. Plant Cell Physiol. 2008, 49, 1580-1588. [CrossRef] [PubMed]

56. Wan, X.; Li, L. Molecular cloning and characterization of a dehydration-inducible cDNA encoding a putative 9-cis-epoxycarotenoid dioxygenase in Arachis hygogaea L. DNA Seq. 2005, 16, 217-223. [CrossRef] [PubMed]

57. Lescot, M.; Dehais, P.; Thijs, G.; Marchal, K.; Moreau, Y.; Van de Peer, Y.; Rouzé, P.; Rombauts, S. PlantCARE, a database of plant cis-acting regulatory elements and a portal to tools for in silico analysis of promoter sequences. Nucleic Acids Res. 2002, 30, 325-327. [CrossRef] [PubMed]

58. Li, X.; Li, X.; Li, M.; Yan, Y.; Liu, X.; Li, L. Dual Function of NAC072 in ABF3-Mediated ABA-Responsive Gene Regulation in Arabidopsis. Front. Plant Sci. 2016, 7, 1075. [CrossRef] [PubMed]

59. Li, X.Y.; Liu, X.; Yao, Y.; Li, Y.H.; Liu, S.; He, C.Y.; Li, J.M.; Lin, Y.Y.; Li, L. Overexpression of Arachis hypogaea AREB1 gene enhances drought tolerance by modulating ROS scavenging and maintaining endogenous ABA content. Int. J. Mol. Sci. 2013, 14, 12827-12842. [CrossRef] [PubMed]

60. Kim, J.M.; To, T.K.; Matsui, A.; Tanoi, K.; Kobayashi, N.I.; Matsuda, F.; Habu, Y.; Ogawa, D.; Sakamoto, T.; Matsunaga, S.; et al. Acetate-mediated novel survival strategy against drought in plants. Nat. Plants 2017, 3, 17097. [CrossRef] [PubMed]

61. Hu, B.; Liu, X.; Hong, L.; Li, L.; Luo, G.Y. Expression and localization of Arachis hypogaea 9-cis epoxycarotenoid dioxygenase 1 (AhNCED1) of peanut under water stress. Biotechnol. Biotechnol. Equip. 2010, 24, 1562-1568. [CrossRef]

62. Liu, S.; Lv, Y.; Wan, X.R.; Li, L.M.; Hu, B.; Li, L. Cloning and expression analysis of cDNAs encoding ABA 8'-hydroxylase in peanut plants in response to osmotic stress. PLoS ONE 2014, 9, e97025. [CrossRef] [PubMed]

63. Liu, S.; Li, M.J.; Su, L.C.; Ge, K.; Li, L.M.; Li, X.Y.; Liu, X.; Li, L. Negative feedback regulation of ABA biosynthesis in peanut (Arachis hypogaea): A transcription factor complex inhibits AhNCED1 expression during water stress. Sci. Rep. 2016, 6, 37943. [CrossRef] [PubMed]

(C) 2018 by the authors. Licensee MDPI, Basel, Switzerland. This article is an open access article distributed under the terms and conditions of the Creative Commons Attribution (CC BY) license (http://creativecommons.org/licenses/by/4.0/). 